\title{
Avaliação de critério de resistência de materiais anisotrópicos aplicado à madeira utilizando ensaios uniaxiais e biaxiais
}

\author{
Nilson Tadeu Mascia(1), Rodrigo Todeschini ${ }^{(2)}$, Elias Antonio Nicolas ${ }^{(3)}$
}

\begin{abstract}
Resumo
A realização de ensaios uniaxiais e biaxiais permite uma melhor compreensão do comportamento mecânico de um material que possui direções principais de resistência e é sem dúvida uma forma que pesquisadores encontraram para obter resultados condizentes com a situação real que uma determinada estrutura apresenta. O presente trabalho teve como objetivo o estudo do critério de resistência proposto por Tsai e Wu, realizando-se ensaios uniaxiais e biaxiais. A avaliação do critério ficou restrita ao ensaio de compressão. Com o ensaio biaxial de compressão, tensões em duas direções principais foram simultaneamente desenvolvidas, utilizando-se um equipamento de ensaio tradicional para uma direção e na direção perpendicular um equipamento tipo alicate desenvolvido para esse fim. $\mathrm{O}$ parâmetro estudado foi o $\mathrm{F}_{12}$, parâmetro que designa a possibilidade da superfície de ruptura ser aberta ou fechada, considerandose que para a segurança de um projeto é imprescindível obter uma superfície fechada para o critério de resistência adotado. Observa-se, além disso, que o parâmetro $F_{12}$ foi obtido no ensaio biaxial de compressão de forma direta. Para as espécies de madeira Pinus elliottii e Goupia glabra obtiveram-se valores experimentais satisfatórios, sendo algumas inconsistências observadas em função da heterogeneidade do material.
\end{abstract}

Palavras-chave: Critérios de Resistência. Critério de Tsai-Wu. Ensaios Uniaxiais e Biaxiais.

1 Professor Associado-Faculdade de Engenharia Civil, Arquitetura e Urbanismo - Unicamp, Av. Albert Einstein, 951 - Campinas /SP. nilson@fec.unicamp.br.

2 Mestre - Engenheiro civil- Petrobras - Engenharia. Av. Alberto Soares Sampaio, 2122 A- Capuava- Mauá/SP. rodrigotodeschini@petrobras.com.br

3 Doutor-Engenheiro civil- Faculdade de Engenharia Civil, Arquitetura e Urbanismo -Unicamp- Av. Albert Einstein, 951 - Campinas /SP. eli_nicolas@yahoo.com.br

http://dx.doi.org/10.5335/rsee.v10i2.1526 


\section{Fundamentos teóricos}

\subsection{Modelo ortotrópico}

O estudo da anisotropia implica no conhecimento da lei constitutiva que rege o material e, conseqüentemente, na determinação do tensor constitutivo e dos elementos presentes nesse tensor.

Nesse contexto, a adequação de um material a um determinado modelo está balizada na existência de eixos de simetria elástica. Nesses eixos há invariância nas propriedades de elasticidade do material. Evidentemente não havendo coincidência entre eixos geométricos e eixos de simetria elástica, o problema recairá em soluções mais complexas e trabalhosas.

Num modelo completamente anisotrópico e elástico existem 81 constantes a se conhecer. Num modelo ortotrópico (três planos de simetria elástica) há 9 elementos presentes no tensor constitutivo,enquanto que num modelo monotrópico há 5 , e assim por diante.

A madeira, devido a sua própria constituição (interna e externa), pode ser considerada ortotrópica. Os eixos de simetria elástica são os eixos longitudinal, L, tangencial,T, e radial, $R$.

No estudo da ortotropia, as diversas teorias que envolvem o estudo dos modelos de rupturas, aplicados aos materiais anisotrópicos, como em Tsai e Wu (1971), baseiam-se não só na anisotropia do material em si, como também, na assimetria das resistências do material. Os tensores $F_{i}$ e $F_{i j}$ cujos coeficientes estão relacionados com certas direções principais e também associados a uma condição de ruptura. No caso dos materiais ortotrópicos, esses possuem três eixos de simetria de resistências, reunidos num tensor de resistência com nove componentes. Na madeira, estão relacionados com as resistências, os eixos de simetria das resistências: L, T e R.

\subsection{Teoria geral de resistência para materiais anisotrópicos}

(Critério de Tsai-Wu)

Para determinar a causa de falha de um material é necessário analisar o estado de tensão a que o material está submetido. Considerando materiais anisotrópicos, deve-se admitir que os modos de ruptura sejam condicionados tanto pelas tensões normais quanto pelas tensões tangenciais, uma vez que as fraturas podem ocorrer em virtude de diferentes conjuntos de tensões que agem sobre o elemento. O critério de Tsai-Wu foi desenvolvido para materiais anisotrópicos, considerando também a assimetria da resistência do material, ou, resistências à tração e compressão diferentes numa mesma direção. A condição geral de ruptura de Tsai-Wu apresenta a seguinte forma:

$$
\mathrm{F}_{\mathrm{i}} \cdot \dot{o}_{\mathrm{i}}+\mathrm{F}_{\mathrm{j}} \cdot \dot{o}_{\mathrm{i}} \cdot \dot{o}_{\mathrm{j}}=1 \quad(\mathrm{i}, \mathrm{j}=1,2,3,4,5,6)
$$


sendo $F_{i}$ os coeficientes de termos lineares de resistência, referentes à ação individual das tensões $o_{i}$ e $F_{i j}$ os coeficientes de termos quadráticos de resistência, referentes à ação conjunta de $o_{i}$ e ó ${ }_{j}$ Assim,em $F_{i}, F_{1}$ é o coeficiente de resistência correspondente à ação isolada a tensão normal $o_{1}$ e o coeficiente $F_{4}$ corresponde à ação isolada da tensão tangencial $\mathrm{ó}_{4}$.

As componentes dos tensores de resistência $F_{i}$ e $F_{i j}$ são expressas pelas matrizes:

$$
\left[\mathrm{F}_{\mathrm{i}}\right]=\left[\begin{array}{ccc}
\mathrm{F}_{1} & \mathrm{~F}_{4} & \mathrm{~F}_{6} \\
& \mathrm{~F}_{2} & \mathrm{~F}_{5} \\
\text { simétrica } & & \mathrm{F}_{3}
\end{array}\right]
$$

$$
\left[\mathrm{F}_{\mathrm{ij}}\right]=\left[\begin{array}{cccccc}
\mathrm{F}_{1} & \mathrm{~F}_{\mathrm{D}} & \mathrm{F}_{\mathrm{B}} & \mathrm{F}_{4} & \mathrm{~F}_{\mathrm{5}} & \mathrm{F}_{6} \\
& \mathrm{~F}_{2} & \mathrm{~F}_{3} & \mathrm{~F}_{\mathfrak{g}} & \mathrm{F}_{\mathrm{g}} & \mathrm{F}_{\mathfrak{b}} \\
& & \mathrm{F}_{3} & \mathrm{~F}_{\mathfrak{3}} & \mathrm{F}_{\mathrm{3}} & \mathrm{F}_{\mathfrak{b}} \\
& & & \mathrm{F}_{4} & \mathrm{~F}_{5} & \mathrm{~F}_{6} \\
& & & & \mathrm{~F}_{5} & \mathrm{~F}_{6} \\
\text { simétrica } & & & & & \mathrm{F}_{6}
\end{array}\right]
$$

No tensor $\mathrm{F}_{\mathrm{ij}}, \mathrm{F}_{11}$ corresponde à ação isolada da tensão $\mathrm{s}_{1}$ e a componente $\mathrm{F}_{12}$ corresponde à ação simultânea das tensões $\mathrm{s}_{1}$ e $\mathrm{s}_{2}$. Nesse caso, os elementos da diagonal principal correspondem à ação isolada das respectivas tensões e os elementos simétricos em relação a essa diagonal são iguais entre si, pois se referem à resistência sob a ação do mesmo par de tensões.

A Equação (1), elaborada por Tsai-Wu (1971), expandida apresenta a seguinte forma:

$$
\begin{gathered}
\mathrm{F}_{1} \cdot \sigma_{1}+\mathrm{F}_{2} \cdot \sigma_{2}+\mathrm{F}_{3} \cdot \sigma_{3}+\mathrm{F}_{4} \cdot \sigma_{4}+\mathrm{F}_{5} \cdot \sigma_{5}+\mathrm{F}_{6} \cdot \sigma_{6}+ \\
+\mathrm{F}_{11} \cdot \sigma_{1}^{2}+2 \mathrm{~F}_{12} \cdot \sigma_{1} \cdot \sigma_{2}+2 \mathrm{~F}_{13} \cdot \sigma_{1} \cdot \sigma_{3}+2 \mathrm{~F}_{14} \cdot \sigma_{1} \cdot \sigma_{4}+2 \mathrm{~F}_{15} \cdot \sigma_{1} \cdot \sigma_{5}+2 \mathrm{~F}_{16} \cdot \sigma_{1} \cdot \sigma_{6}+ \\
+\mathrm{F}_{22} \cdot \sigma_{2}^{2}+2 \mathrm{~F}_{23} \cdot \sigma_{2} \cdot \sigma_{3}+2 \mathrm{~F}_{24} \cdot \sigma_{2} \cdot \sigma_{4}+2 \mathrm{~F}_{25} \cdot \sigma_{2} \cdot \sigma_{5}+2 \mathrm{~F}_{26} \cdot \sigma_{2} \cdot \sigma_{6}+ \\
+\mathrm{F}_{33} \cdot \sigma_{3}^{2}+2 \mathrm{~F}_{34} \cdot \sigma_{3} \cdot \sigma_{4}+2 \mathrm{~F}_{35} \cdot \sigma_{3} \cdot \sigma_{5}+2 \mathrm{~F}_{36} \cdot \sigma_{3} \cdot \sigma_{6}+ \\
+\mathrm{F}_{44} \cdot \sigma_{4}^{2}+2 \mathrm{~F}_{45} \cdot \sigma_{4} \cdot \sigma_{5}+2 \mathrm{~F}_{46} \cdot \sigma_{4} \cdot \sigma_{6}++\mathrm{F}_{55} \cdot \sigma_{5}^{2}+2 \mathrm{~F}_{56} \cdot \sigma_{5} \cdot \sigma_{6}+\mathrm{F}_{66} \cdot \sigma_{6}^{2}=1
\end{gathered}
$$

Para um material anisotrópico existem 6 elementos independentes do tensor $\mathrm{F}_{\mathrm{i}} \mathrm{e}$ 21 elementos independentes do tensor $\mathrm{F}_{\mathrm{ij}}$. 
As várias características do critério de resistência de Tsai-Wu (1971) são as seguintes: é uma equação escalar e automaticamente invariante. As interações entre todos os componentes de tensões são independentes das propriedades do material; as componentes de resistência são expressas em um tensor, suas relações de transformação e os invariantes associados são bem estabelecidos; as propriedades de simetria do tensor de resistência e o número de componentes nulos e independentes podem ser rigorosamente determinados, da mesma forma que outras propriedades dos materiais anisotrópicos, tal como a matriz de elasticidade; sendo invariante, o critério de resistência é válido para todos os sistemas de coordenadas.

Embora muitas superfícies possam ser representadas por um polinômio na forma da Equação (4), nem todas as superfícies são superfícies de ruptura admissíveis. A superfície de ruptura não pode ser imaginária nem ser uma superfície aberta. Para satisfazer essas condições algumas restrições para os coeficientes $F_{i}$ e $F_{i j}$ são necessárias.

Para o estado plano de tensões (plano 1-2), observa-se que a superfície de ruptura é tri-dimensional, pois há três componentes de tensões independentes, $\sigma_{1}, \sigma_{2}$ e $\sigma_{4}$ A condição de estabilidade, necessária e suficiente, para o critério de ruptura para um material de resistência finita é representada pela Equação (5):

$$
\mathrm{F}_{\mathrm{ii}} \cdot \mathrm{F}_{\mathrm{jj}}-\mathrm{F}_{\mathrm{ij}}^{2} \geq 0
$$

com $\mathrm{F}_{\mathrm{ii}}$ representa os termos da diagonal principal. Para ter significado físico, todos os termos da diagonal principal devem ser positivos; os termos de fora da diagonal principal podem ser negativos ou positivos dependendo da natureza de interação, mas suas magnitudes são restringidas pela Equação (5). Geometricamente essa inequação assegura que a superfície de ruptura interceptará cada um dos eixos coordenados de tensões. A forma da superfície será um elipsóide. Se essa inequação não for respeitada, a superfície de ruptura se torna aberta.

\subsection{Determinação dos parâmetros de resistência do critério de Tsai-Wu para materiais ortotrópicos}

Através de ensaios de tração e compressão paralela e perpendicular às fibras, e ensaios de cisalhamento paralelo às fibras é possível determinar quase todos os parâmetros do critério de Tsai-Wu. A Tabela 1 contêm os valores dos coeficientes $F_{i}(i=1,2$, 3) e $\mathrm{F}_{\mathrm{ii}}(\mathrm{i}=1$ a 6$)$.

Os valores de $f_{t i}, f_{v i} f_{c i}$ são respectivamente as resistências à tração, à compressão e ao cisalhamento para uma determinada direção principal i, as quais são obtidas através dos ensaios uniaxiais de tração, compressão e cisalhamento. 
Tabela 1 - Coeficientes $F_{i}$ e $F_{i i}$.

\begin{tabular}{c|c|c}
\hline $\mathrm{F}_{1}=\frac{1}{\mathrm{f}_{\mathrm{t} 1}}-\frac{1}{\mathrm{f}_{\mathrm{c} 1}}$ & $\mathrm{~F}_{2}=\frac{1}{\mathrm{f}_{\mathrm{t} 2}}-\frac{1}{\mathrm{f}_{\mathrm{c} 2}}$ & $\mathrm{~F}_{3}=\frac{1}{\mathrm{f}_{\mathrm{t} 3}}-\frac{1}{\mathrm{f}_{\mathrm{c} 3}}$ \\
\hline $\mathrm{F}_{11}=\frac{1}{\mathrm{f}_{\mathrm{t} 1} \cdot \mathrm{f}_{\mathrm{c} 1}}$ & $\mathrm{~F}_{2}=\frac{1}{\mathrm{f}_{\mathrm{t} 2} \cdot \mathrm{f}_{\mathrm{c} 2}}$ & $\mathrm{~F}_{3}=\frac{1}{\mathrm{f}_{\mathrm{t} 3} \cdot \mathrm{f}_{\mathrm{c} 3}}$ \\
\hline $\mathrm{F}_{44}=\frac{1}{\mathrm{f}_{\mathrm{v} 4}^{2}}$ & $\mathrm{~F}_{55}=\frac{1}{\mathrm{f}_{\mathrm{v} 5}^{2}}$ & $\mathrm{~F}_{66}=\frac{1}{\mathrm{f}_{\mathrm{v} 6}^{2}}$ \\
\hline
\end{tabular}

Os seis casos de combinações de tensões sugeridos por Tsai e Wu (1971) para a determinação do coeficiente de interação $\mathrm{F}_{12}$ estão apresentados na Figura1. Na Tabela 2 mostra-se a expressão de $\mathrm{F}_{12}$ para os seis casos, de acordo com Tsai e Wu (1971). Cada um dos casos apresenta $\left|\boldsymbol{\sigma}_{1} / \mathbf{a}_{\mathbf{2}}\right|=\mathbf{1}$.

Na Figura 1 e na Tabela 2, observa-se que t indica tensão de tração, p de compressão, Ut tensão de tração inclinada, Up de compressão inclinada, Vp tensão positiva de cisalhamento e Vn tensão negativa de cisalhamento.

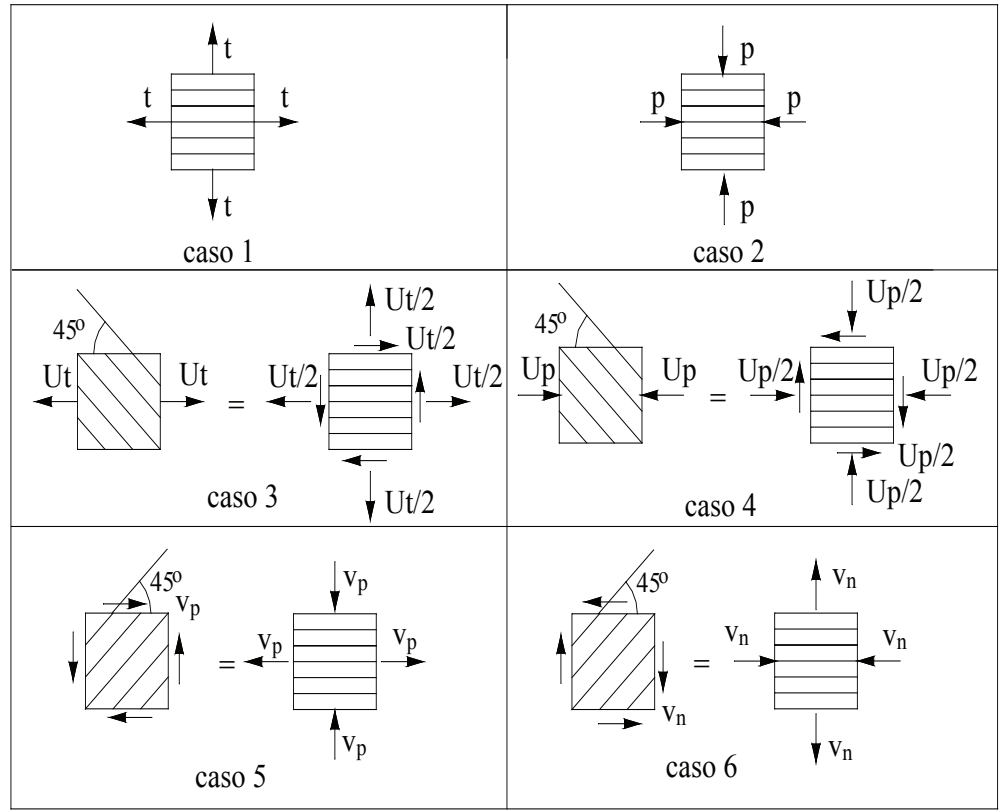

Figura 1 - Ensaio biaxiais e uniaxiais (casos 1 a 6 ). 
Outras expressões para determinação do coeficiente $\mathrm{F}_{12}$ sugerido por Gol'denblat-Kopnov (1965), Hoffman (1967), Cowin (1979) e Liu (1984) estão listados na Tabela 3.

Tabela 2 - Coeficiente de interação de tensões $F_{12}$ com diferentes combinações detensões.

\begin{tabular}{l|c|c}
\hline Caso & Tensões $\left(\sigma_{1}, \sigma_{2}, \sigma_{12}\right)$ & $F_{12}$ \\
\hline 1 & $(\mathrm{t}, \mathrm{t}, 0)$ & $\frac{1}{2 \mathrm{t}^{2}} \cdot\left[1-\mathrm{t} \cdot\left(\mathrm{F}_{1}+\mathrm{F}_{2}\right)-\mathrm{t}^{2} \cdot\left(\mathrm{F}_{1}+\mathrm{F}_{2}\right)\right]$ \\
2 & $(-\mathrm{p},-\mathrm{p}, 0)$ & $\frac{1}{2 \mathrm{p}^{2}} \cdot\left[1+\mathrm{p} \cdot\left(\mathrm{F}_{1}+\mathrm{F}_{2}\right)-\mathrm{p}^{2} \cdot\left(\mathrm{F}_{1}+\mathrm{F}_{2}\right)\right]$ \\
3 & $\left(\mathrm{U}_{\mathrm{t}} / 2, \mathrm{U}_{\mathrm{t}} / 2, \mathrm{U}_{\mathrm{t}} / 2\right)$ & $\frac{2}{\mathrm{U}_{\mathrm{t}}^{2}} \cdot\left[1-\frac{\mathrm{U}_{\mathrm{t}}}{2} \cdot\left(\mathrm{F}_{1}+\mathrm{F}_{2}\right)-\frac{\mathrm{U}_{\mathrm{t}}^{2}}{4} \cdot\left(\mathrm{F}_{1}+\mathrm{F}_{2}+\mathrm{F}_{4}\right)\right]$ \\
& $\left(-\mathrm{U}_{\mathrm{p}} / 2,-\mathrm{U}_{\mathrm{p}} / 2,-\mathrm{U}_{\mathrm{p}} / 2\right)$ & $\frac{2}{\mathrm{U}_{\mathrm{p}}^{2}} \cdot\left[1+\frac{\mathrm{U}_{\mathrm{p}}}{2} \cdot\left(\mathrm{F}_{1}+\mathrm{F}_{2}\right)-\frac{\mathrm{U}_{\mathrm{p}}^{2}}{4} \cdot\left(\mathrm{F}_{1}+\mathrm{F}_{2}+\mathrm{F}_{4}\right)\right]$ \\
& $\left(\mathrm{v}_{\mathrm{p}},-\mathrm{v}_{\mathrm{p}}, 0\right)$ & $-\frac{1}{2 \mathrm{v}_{\mathrm{p}}^{2}} \cdot\left[1-\mathrm{v}_{\mathrm{p}} \cdot\left(\mathrm{F}_{1}-\mathrm{F}_{2}\right)-\mathrm{v}_{\mathrm{p}}^{2} \cdot\left(\mathrm{F}_{1}+\mathrm{F}_{2}\right)\right]$ \\
5 & & $-\frac{1}{2 \mathrm{v}_{\mathrm{n}}^{2}} \cdot\left[1+\mathrm{v}_{\mathrm{n}} \cdot\left(\mathrm{F}_{1}-\mathrm{F}_{2}\right)-\mathrm{v}_{\mathrm{n}}^{2} \cdot\left(\mathrm{F}_{1}+\mathrm{F}_{2}\right)\right]$ \\
\hline
\end{tabular}

Tabela 3 - Expressões do coeficiente $\mathrm{F}_{12}$ de acordo alguns pesquisadores.

\begin{tabular}{l|c}
\hline \multicolumn{1}{c|}{ Pesquisador } & $\mathrm{F}_{12}$ \\
\hline Hoffman & $\frac{1}{2 \cdot \mathrm{f}_{\mathrm{t} 1} \cdot \mathrm{f}_{\mathrm{cl}}}$ \\
\hline Liu & $\frac{1}{\sqrt{\mathrm{f}_{\mathrm{t} 1} \cdot \mathrm{f}_{\mathrm{c} 1} \cdot \mathrm{f}_{\mathrm{t} 2} \cdot \mathrm{f}_{\mathrm{c} 2}}}-\frac{1}{2 \cdot \mathrm{f}_{\mathrm{v}}^{2}}$ \\
& $\frac{1}{2} \cdot\left(\frac{1}{\mathrm{f}_{\mathrm{t} 1} \cdot \mathrm{f}_{\mathrm{c} 2}}+\frac{1}{\mathrm{f}_{\mathrm{c} 1} \cdot \mathrm{f}_{\mathrm{t} 2}}-\frac{1}{\mathrm{f}_{\mathrm{v}}^{2}}\right)$ \\
\hline
\end{tabular}




\subsection{Ensaios Biaxiais}

A realização de ensaios uniaxiais e biaxiais permite uma análise geral do comportamento das propriedades mecânicas do material simultaneamente em suas direções principais e é, sem dúvida, a forma que engenheiros pesquisadores encontraram para obter resultados que permitem a calibração dos modelos teóricos adotados.

Uma das grandes dificuldades enfrentadas nesses tipos de ensaios é a elaboração de um maquinário que possa corresponder fielmente às situações de carregamento em uma estrutura real, assim como obter dispositivos eficientes para a fixação do corpo de - prova. $\mathrm{O}$ alicate desenvolvido na realização do ensaio biaxial de compressão para a madeira é muito sensível à calibração.

Além da madeira, pode-se observar a existência de ensaios biaxiais em outros tipos de materiais. $\mathrm{O}$ modelo ortotrópico é comumente utilizado nos materiais para esses tipos de ensaios. Como exemplo, pode-se observar uma aplicação do critério de Tsai-Wu em estimativas de resistências aplicadas à madeira e ao papelão.

Nesse sentido, Hasebe and Usuki (1989) aplicaram a teoria geral de resistência para materiais anisotrópicos como a madeira, considerando o estado plano de tensões, na qual a direção 1 paralela às fibras da madeira e a direção 2 perpendicular às fibras, obtendo a equação:

$$
\mathrm{F}_{1} \cdot \sigma_{1}+\mathrm{F}_{2} \cdot \sigma_{2}+\mathrm{F}_{11} \cdot \sigma_{1}^{2}+\mathrm{F}_{22} \cdot \sigma_{2}^{2}+2 \mathrm{~F}_{12} \cdot \sigma_{1} \cdot \sigma_{2}+\mathrm{F}_{44} \cdot \sigma_{4}^{2}=1
$$

Como a influência da tensão ( $\sigma_{2}$, na direção perpendicular às fibras, é pequena, pode ser desprezada. Desse modo a Equação (6) se reduz a forma:

$$
\mathrm{F}_{1} \cdot \sigma_{1}+\mathrm{F}_{11} \cdot \sigma_{1}^{2}+\mathrm{F}_{44} \cdot \sigma_{4}^{2}=1
$$

A madeira utilizada foi o "Japanese Cedar" e foram realizados ensaios uniaxiais de tração, compressão e cisalhamento em corpos - de -prova. Com os resultados dos ensaios foram determinados os coeficientes da Eq. (7) e foram construídas as superfícies de ruptura.

Eberhardsteiner (2002) realizou ensaios biaxiais em madeira de "Spruce" com o objetivo de obter as superfícies de Tsai-Wu e os modos de ruptura. Por meio da Figura 2 observa-se o equipamento de testes e o corpo-de-prova da madeira, na qual a espessura do corpo-de-prova é muito pequena em relação às outras dimensões, evidenciando assim, um estado plano de tensões. 

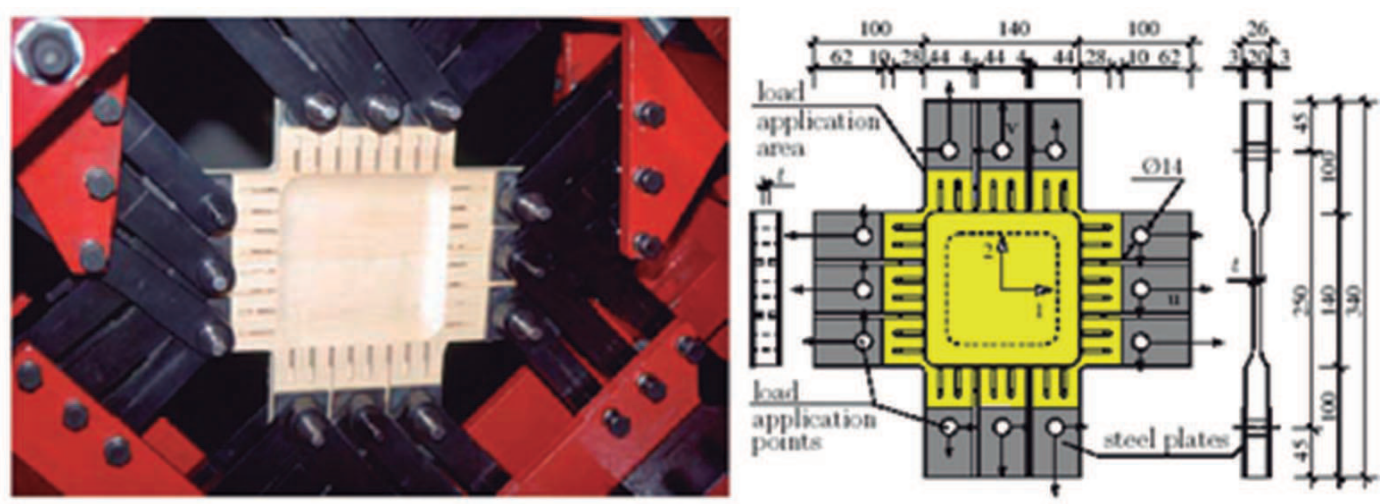

Figura 2 - Equipamento biaxial e detalhe do corpo - de - prova, com dimensões em mm. Fonte: Eberhardsteiner (2002)

Suhling (1985) realizou ensaios biaxiais no papelão e comparou os resultados com a teoria de ruptura de Tsai-Wu. Na Tabela 4 estão os resultados das propriedades mecânicas do material.

Tabela 4 - Propriedades mecânicas do papelão.

\begin{tabular}{c|c}
\hline Propriedades & Valores (MPa) \\
\hline $\mathrm{f}_{\mathrm{t} 0}$ & 55,90 \\
$\mathrm{f}_{\mathrm{t} 90}$ & 20,50 \\
$\mathrm{f}_{\mathrm{c} 0}$ & 30,80 \\
$\mathrm{f}_{\mathrm{c} 90}$ & 12,70 \\
$\mathrm{f}_{\mathrm{v}}$ & 16,60 \\
\hline
\end{tabular}

Com a Tabela 4 foram calculados os parâmetros do critério de resistência, com $F_{12}$ determinado com: $-\sqrt{F_{11} \cdot F_{22}} \leq F_{12} \leq \sqrt{F_{11} \cdot F_{22}}$ ou $-15 \cdot 10^{-4} \mathrm{MPa}^{-2} \leq F_{12} \leq 15 \cdot 10^{-4} \mathrm{MPa}^{-2}$.

Os valores utilizados acima são apenas uma pequena faixa dos valores possíveis do parâmetro $\mathrm{F}_{12}$. Isso demonstra a grande sensibilidade do critério de resistência quando utilizado na comparação com ensaios biaxiais.

Pode-se citar, também, o trabalho de Alvin (2003), em que o principal objetivo foi avaliar as propriedades mecânicas dos tecidos estruturais e para isso foi desenvolvido um arranjo de ensaio que permite a aplicação das forças de tração (semelhante ao caso 1, Figura 4,proposto por Tsai-Wu). Foram realizados ensaios uniaxiais e biaxiais para esses tipos de tecidos. Para os ensaios uniaxiais os tecidos estruturais foram ensaiados com o carregamento paralelo às fibras (tramas e urdumes) e para os ensaios biaxiais as amostras foram submetidas a esforços ortogonais de tração, simultaneamente e de mesma intensidade, alinhados com as direções da trama e do urdume. 
Note-se a grande semelhança na realização dos ensaios uniaxiais do tecido com a madeira, devido a aplicação da carga ser paralela e perpendicular às fibras de crescimento. Já nos ensaios biaxiais tem-se o ensaio de tração para o tecido e o ensaio de compressão para a madeira, pois o tecido só apresenta estrutura resistente ao ser tracionado, ao contrário da madeira que pode ser tracionada e comprimida por ter estrutura mais rígida.

Na Unicamp-Brasil pesquisas sobre o critério de Tsai-Wu foram desenvolvidas, a partir de resultados de ensaios uniaxiais e biaxiais. Pode-se citar: Nicolas (2006); Mascia, Nicolas and Todeschini (2007); Mascia, Nicolas and Sverzut (2007) e Todeschini, Mascia and Nicolas (2008).

\section{Ensaio de compressão biaxial}

Conforme explicitado anteriormente, tem-se a equação geral para materiais anisotrópicos, que através das considerações de simetria e do modelo ortotrópico e desenvolvida para o caso bidimensional 1-2, apresenta a seguinte forma:

$$
\mathrm{F}_{1} \cdot \sigma_{1}+\mathrm{F}_{2} \cdot \sigma_{2}+\mathrm{F}_{11} \cdot \sigma_{1}^{2}+\mathrm{F}_{22} \cdot \sigma_{2}^{2}+2 \mathrm{~F}_{12} \cdot \sigma_{1} \cdot \sigma_{2}+\mathrm{F}_{44} \cdot \sigma_{4}^{2}=1
$$

Pode-se obter os valores de $\mathrm{F}_{\mathrm{i}}$ e $\mathrm{F}_{\mathrm{ij}}$ através de resistências mostradas, por exemplo, na Tabela 1. Observa-se que os valores de $f_{t i} f_{c i}{ }^{e} f_{v 4}$ são as tensões de ruptura nas direções 1 e 2 da peça, obtidas nos ensaios uniaxiais de tração, compressão e cisalhamento. Os índices 1 e 2 correspondem às direções principais da peça, respectivamente, paralela e perpendicular à direção das fibras da madeira.

Nota-se que, no ensaio de compressão biaxial, o fator $\mathrm{F}_{12}$ pode ser isolado na Eq. (8)

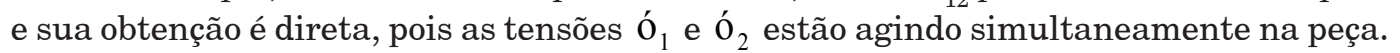

A escolha do arranjo para o ensaio de compressão biaxial foi devido à dificuldade de se elaborar dispositivos de fixação da peça de madeira para as situações de tração na peça, sendo mais simples e mais econômico comprimir a peça de madeira através de uma máquina uniaxial de compressão, junto com um alicate de compressão desenvolvido para esse fim.

O alicate de compressão atua na direção perpendicular (2) às fibras de crescimento da madeira, enquanto que na direção paralela (1) às fibras a força de compressão foi aplicada pela máquina de ensaio universal Emic, de $300 \mathrm{kN}$ de capacidade.

A Figura 3 mostra o equipamento de compressão axial (alicate), e o equipamento usado para o ensaio de compressão biaxial, desenvolvido e construído pelo Centro de Tecnologia (CT) da Unicamp. Nesse ensaio foi utilizada uma máquina de ensaio universal Mohr-Federhaff com capacidade de $400 \mathrm{kN}$. O alicate foi confeccionado com o aço CGR Sae 1045 com espessura de 2" e tensão de escoamento de $310 \mathrm{MPa}$. Para a aplicação da carga na máquina de compressão transversal (alicate) foi utilizado um cilindro Enerpac Desf C101, calibrado pela célula de carga HBM Typ z12, \#H0748, certificado Inmetro/Dimci 1064/2002. O cilindro foi posicionado entre as hastes maiores do alicate, 
aplicando uma força na tentativa de abri-lo. Desse modo, houve transferência de esforços para o ponto de contato entre o alicate e a madeira. Para o sistema de aquisição dos dados da máquina de compressão foi utilizada uma ponte HBM 6 canais, amplificador nº 22648, indicador no 98860 para o cilindro Enerpac e uma ponte HBM DK 8, canal 11 para a célula de carga HBM.

O ensaio de compressão biaxial é iniciado pela aplicação de uma força de compressão no corpo-de- prova de madeira (cubo de $4 \mathrm{~cm}$ de aresta) utilizando-se a máquina de compressão axial (alicate), sem atingir a ruptura nessa direção. Na sequência aplicou-se o carregamento utilizando-se a máquina universal de ensaios até atingir a ruptura, na qual se obtêm o par de tensões $\left(\sigma_{1}\right.$ e $\left.\sigma_{2}\right)$ com os vários pares de tensões obtidos no ensaio construiu-se o gráfico com os pontos no gráfico $\sigma_{1} \times \sigma_{2}$, verificando a hipótese do critério de resistência.

Ao todo foram ensaiados 32 corpos - de - prova da madeira Pinus elliottii e 62 da madeira Goupia glabra (Cupiúba).

Para a aplicação da força no equipamento de compressão foi utilizado um macaco hidráulico. Foram utilizados oito extensômetros elétricos (KFG-5-120-C1-11 da Kyowa) no equipamento de compressão axial, adotando um sistema de ponte completa dupla. De acordo com a deformação do equipamento sob aplicação de carga, pode-se medir a força de compressão aplicada.
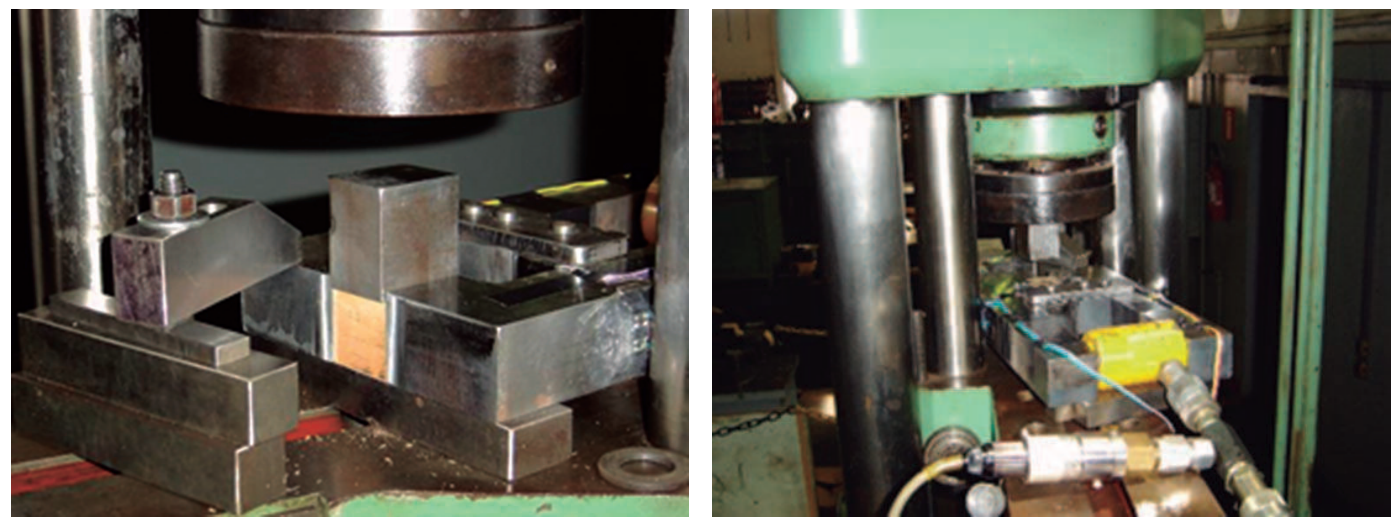

Figura 3 - Ensaio de compressão biaxial.

\section{Análise dos ensaios biaxiais de compressão}

A partir de ensaios uniaxiais de compressão das espécies de madeira estudadas (Pinus elliottii e Goupia glabra) serão apresentados seus respectivos resultados.

Para o cálculo das outras resistências da espécie Pinus elliottii, a partir da resistência à compressão, foram obtidas de acordo com relações da ABNT-NBR 7190 (1997) para valores característicos: $\mathrm{f}_{\mathrm{cl}}=\mathrm{f}_{\mathrm{co}}=32,0 \mathrm{MPa}$. 
O valor de $\mathrm{f}_{\mathrm{t} 0}$ foi obtido da seguinte relação da ABNT- NBR 7190 (1997) item 5.3.3: $\frac{\mathrm{f}_{\mathrm{c} 0}}{\mathrm{f}_{\mathrm{t} 0}}=0,77$, resultando em $\mathrm{f}_{\mathrm{t} 0}=\mathrm{f}_{\mathrm{t} 1}=41,56 \mathrm{MPa}$. Já $\mathrm{f}_{\mathrm{c} 90}$ foi obtido da seguinte relação, $\frac{f_{c 90}}{f_{c 0}}=0,25$. Assim: $f_{c 90}=f_{c 2}=8,0 \mathrm{MPa}$. O valor de $f_{v}$ foi estimado por $\frac{f_{v 0, k}}{f_{c 0, k}}=0,15$ ou seja, $\mathrm{f}_{\mathrm{v}}=\mathrm{f}_{\mathrm{v} 4}=4,8 \mathrm{MPa}$ e finalmente $\mathrm{f}_{\mathrm{t} 90}=\mathrm{f}_{\mathrm{t} 2}=2,5 \mathrm{MPa}$ via Talela E.3 da ABNT-NBR 7190 (1997) por ser valores médios da espécie Pinus elliottii.

Com os valores das resistências são calculados os parâmetros de resistência da Tabela 1 e ainda os limites para $\mathrm{F}_{12}$. Seguem-se os resultados na Tabela 5.

Com os valores da Tabela 5, pode-se substituí-los na Equação (8) e deixá-la em função apenas das tensões $\sigma_{1}$ e $\sigma_{2}$. A tensão de cisalhamento $\sigma_{4}$ é perpendicular ao plano 1-2 e, portanto seu valor será adotado igual à zero. Assim tem-se:

$$
\begin{aligned}
& \left(-7,19 \times 10^{-3}\right) \cdot \sigma_{1}+\left(2,75 \times 10^{-1}\right) \cdot \sigma_{2}+\left(7,52 \times 10^{-4}\right) \cdot \sigma_{1}^{2}+\left(5,0 \times 10^{-2}\right) \cdot \sigma_{2}^{2}+ \\
& +2 \mathrm{~F}_{12} \cdot \sigma_{1} \cdot \sigma_{2}+\left(4,34 \times 10^{-2}\right) \cdot \sigma_{4}^{2}=1
\end{aligned}
$$

Nos ensaios biaxiais de compressão, as tensões ó ${ }_{1}$ e ó ${ }_{2}$ apresentam sinais negativos e devem ser considerados na equação. A seguir na Tabela 6 são apresentados, apenas para a espécie de madeira Pinus elliottii, os resultados dos ensaios biaxiais de compressão com o cálculo do parâmetro $\mathrm{F}_{12}$ btido através da Equação 9.

Tabela 5 - Parâmetros de resistência e limites de $\mathrm{F}_{12}$ da madeira Pinus elliottii.

\begin{tabular}{c|c|c}
\hline Parâmetro & Expressão & Valor \\
\hline$F_{1}$ & $\frac{1}{\mathrm{f}_{\mathrm{t} 1}}-\frac{1}{\mathrm{f}_{\mathrm{c} 1}}$ & $-7,19 \times 10^{-3} \mathrm{MPa}^{-1}$ \\
\hline $\mathrm{F}_{2}$ & $\frac{1}{\mathrm{f}_{\mathrm{t} 2}}-\frac{1}{\mathrm{f}_{\mathrm{c} 2}}$ & $2,75 \times 10^{-1} \mathrm{MPa}^{-1}$ \\
\hline $\mathrm{F}_{11}$ & $\frac{1}{\mathrm{f}_{\mathrm{t} 1}} \times \frac{1}{\mathrm{f}_{\mathrm{c} 1}}$ & $7,52 \times 10^{-4} \mathrm{MPa}^{-2}$ \\
\hline $\mathrm{F}_{22}$ & $\frac{1}{\mathrm{f}_{\mathrm{t} 2}} \times \frac{1}{\mathrm{f}_{\mathrm{c} 2}}$ & $5,0 \times 10^{-2} \mathrm{MPa}^{-2}$ \\
\hline $\mathrm{F}_{44}$ & $\frac{1}{\mathrm{f}_{\mathrm{v} 4}{ }^{2}}$ & $4,34 \times 10^{-2} \mathrm{MPa}^{-2}$ \\
\hline Limite de $\mathrm{F}_{12}$ & $\pm \sqrt{\mathrm{F}_{11} \times \mathrm{F}_{22}}$ & $\pm 6,13 \times 10^{-3} \mathrm{MPa}^{-2}$ \\
\hline
\end{tabular}


Tabela 6 - Resultados do ensaio biaxial de compressão e valores de $F_{12}$ da madeira Pinus.

\begin{tabular}{c|c|c|c|c}
\hline Carga Vertical (daN) & Carga no Alicate (daN) & $\begin{array}{c}\mathrm{O}_{1} \\
(\mathrm{MPa})\end{array}$ & $\begin{array}{c}\mathrm{O}_{2} \\
(\mathrm{MPa})\end{array}$ & $\mathrm{F}_{12}\left(\mathrm{MPa}^{-2}\right)$ \\
\hline 3850 & 517 & $-24,06$ & $-3,23$ & 0,00488 \\
4150 & 517 & $-25,94$ & $-3,23$ & 0,00402 \\
4450 & 1019 & $-27,81$ & $-6,37$ & $-0,000166$ \\
4400 & 1016 & $-27,50$ & $-6,35$ & $-0,000104$ \\
4750 & 1034 & $-29,69$ & $-6,46$ & $-0,000486$ \\
4000 & 323 & $-25,00$ & $-2,02$ & 0,00695 \\
4450 & 352 & $-27,81$ & $-2,20$ & 0,00475 \\
4200 & 776 & $-26,25$ & $-4,85$ & 0,00177 \\
4900 & 1163 & $-30,63$ & $-7,27$ & $-0,001280$ \\
\hline
\end{tabular}

Nota-se variações nos valores do parâmetro $\mathrm{F}_{12}$, mostrando a grande variabilidade do mesmo. Mesmo com as variações observadas do parâmetro, verifica-se que eles obedecem à condição limite, Equação (5), apresentando uma superfície fechada.

Os valores descartados de $\mathrm{F}_{12}$ apresentaram-se elevados, pois ao se isolar esse fator na Equação (8) tem-se a divisão por zero pelo fato das tensões estarem se multiplicando. Assim, caso $\sigma_{1}$ ou $\sigma_{2}$ apresentem o valor zero, o parâmetro $\mathrm{F}_{12}$ crescerá, sendo desconsiderado na análise.

Tabela 7 - Médias dos valores de $\mathrm{F}_{12}$ (Pinus).

\begin{tabular}{c}
\hline Médias de $\mathrm{F}_{12}\left(\mathrm{MPa}^{-2}\right)$ \\
\hline $0,001572(\mathrm{I})$ \\
$-0,001670$ (II) \\
0,005013 (III) \\
$-0,000252$ (IV) \\
\hline
\end{tabular}

Valores limites de $\mathrm{F}_{12}$ para comparação:

Tabela 8 - Valores limites de $\mathrm{F}_{12}$ (Pinus).

\begin{tabular}{c}
\hline Limites de $\mathrm{F}_{12}\left(\mathrm{MPa}^{-2}\right)$ \\
\hline 0,00613 \\
$-0,00613$ \\
\hline
\end{tabular}

Ao se comparar os valores da Tabela 7 e Tabela 8, verifica-se que todos estão dentro do intervalo e, portanto apresentam a equação de uma elipse no plano. Assim, para cada valor de $\mathrm{F}_{12}$, serão montadas curvas, provenientes da Equação (9) e que podem ser observadas na Figura 4. Para uma melhor análise dos resultados, obtiveram-se médias para $\mathrm{F}_{12}$. Esses valores substituídos na Equação (9) resultam na equação do critério de resistência de Tsai-Wu. 
Avaliação de critério de resistência de materiais anisotrópicos aplicado à madeira...

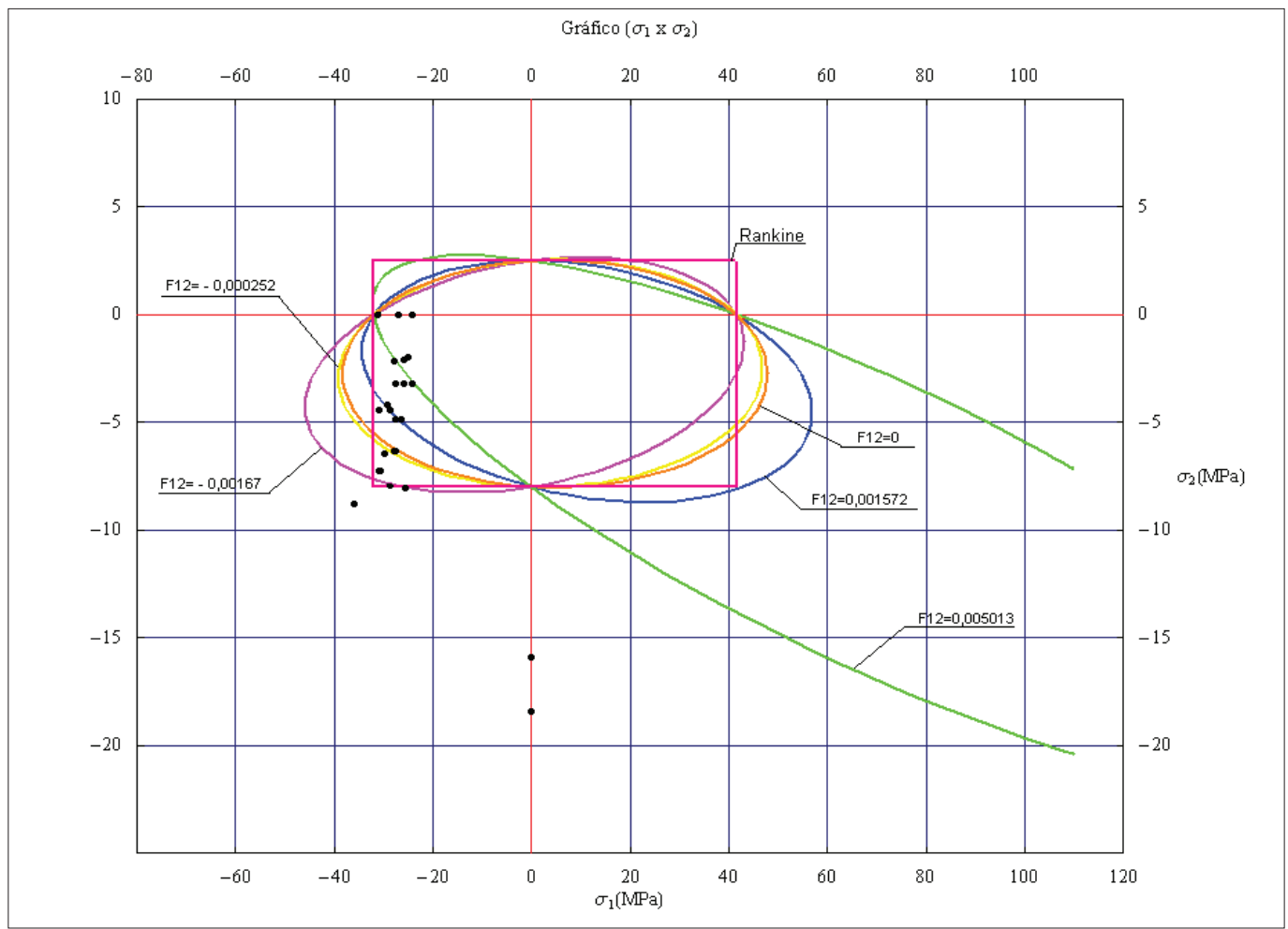

Figura 4 - Curvas provenientes da Equação. (9) com valores de $F_{12}$ (Pinus).

Analogamente todo esse procedimento é feito para a espécie de madeira Goupia glabra - Cupiúba.

Tabela 9 - Médias dos valores de $F_{12}$ (Cupiúba).

\begin{tabular}{c}
\hline Médias de $\mathrm{F}_{12}\left(\mathrm{MPa}^{-2}\right)$ \\
0,000486 \\
0,000803 \\
0,000192 \\
$-0,000056$ \\
0,000026 \\
0,000097 \\
$-0,000330$ \\
0,001692 \\
0 \\
\hline
\end{tabular}


Valores limites de $\mathrm{F}_{12}$ para comparação:

Tabela 10 - Valores Limites de $\mathrm{F}_{12}$ (Cupiúba).

$$
\begin{gathered}
\text { Limites de } F_{12}\left(\mathrm{MPa}^{-2}\right) \\
0,00159 \\
-0,00159
\end{gathered}
$$

Ao se comparar os valores da Tabela 9 com os valores limites da Tabela 10, verifica-se que apenas o valor $1,692 \times 10^{-3} \mathrm{MPa}$ está fora do intervalo, evidenciando provável superfície de ruptura aberta. Assim, para cada valor de $\mathrm{F}_{12}$, pode-se montar curvas, provenientes da Equação (10) e que estão representadas na Figura 5. Na Figura 4 e na Figura 5 utilizou-se como referência o critério de Rankine (Chen and Salleb, 1982).

$$
\begin{aligned}
\left(-3,51 \times 10^{-3}\right) \cdot \sigma_{1}+\left(1,65 \times 10^{-1}\right) \cdot \sigma_{2}+( & \left.2,36 \times 10^{-4}\right) \cdot \sigma_{1}^{2}+\left(1,07 \times 10^{-2}\right) \cdot \sigma_{2}^{2}+ \\
& +2 \mathrm{~F}_{12} \cdot \sigma_{1} \cdot \sigma_{2}+\left(2,56 \times 10^{-3}\right) \cdot \sigma_{4}^{2}=1
\end{aligned}
$$

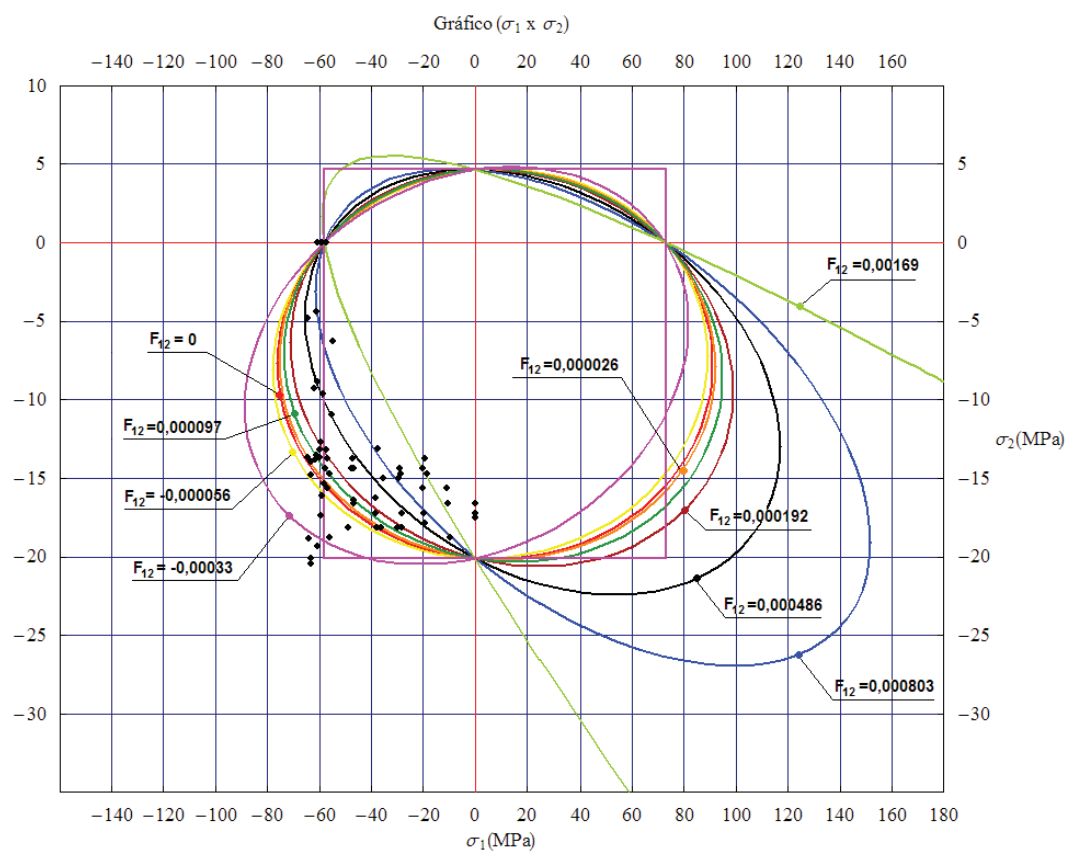

Figura 5 - Curvas provenientes da Equação (9) com valores de $F_{12}$ (Cupiúba). 


\section{Modelagem numérica do corpo-de-prova}

nessa modelagem foi considerada a madeira da espécie Pinus elliottii com as seguintes propriedades físicas e mecânicas, de acordo com Ferreira (2003): densidade com $15 \%$ de umidade: $480 \mathrm{~kg} / \mathrm{m}^{3}$;resistência à compressão paralela: $\mathrm{f}_{\mathrm{c} 0}=18,5 \mathrm{MPa}$ para madeira verde e $\mathrm{f}_{\mathrm{c} 0}=31,5$ MPacom $15 \%$ de umidade e módulo de elasticidade longitudinal, madeira verde $\mathrm{E}_{\mathrm{c}}=8846 \mathrm{MPa}$.

Para a realização da modelagem, utilizou-se o software SAP 2000 (2010) que é baseado no método dos elementos finitos. Nessa análise simulou-se um corpo-de-prova cúbico com arestas de $4 \mathrm{~cm}$ e com as disposições das fibras paralelas à direção da aplicação da carga da prensa universal, ou seja, a $0^{\circ}$. A aplicação do alicate se dá na direção perpendicular às fibras, ou seja, a $90^{\circ}$.

O esquema estático adotado visou simular a situação real na qual o corpo- de-prova se encontrava (Figura 6). Como o corpo-de-prova era simplesmente apoiado, considerou-se esse ponto de apoio com apoio móvel nas direções 1 (ou x) e 2 (ou y), apenas restringiu-se a direção 3 (ou z). Como o alicate pressionava na direção perpendicular de ambos os lados, não houve necessidade de restringir essa direção (no caso, direção 2). Logo, obteve-se o modelo sólido indicado na Figura 6:

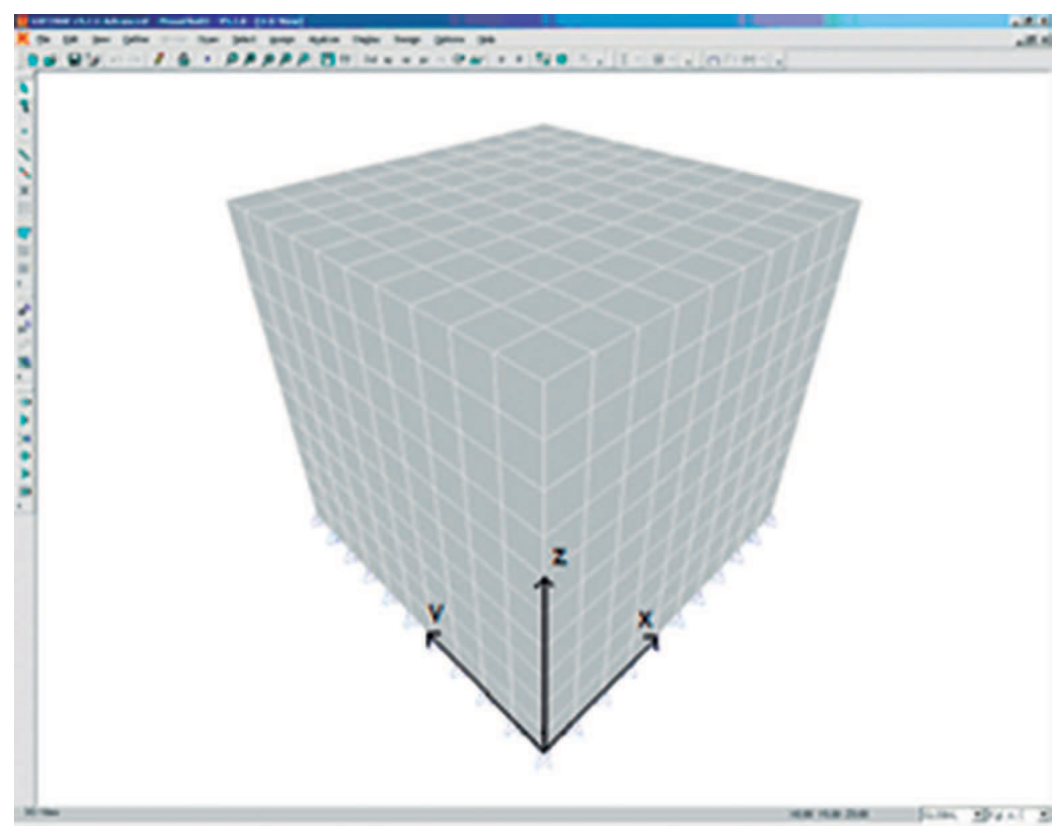

Figura 6 - Corpo-de-prova modelado no SAP2000. 
Observa-se que a madeira possui comportamento anisotrópico, mas por simplificação no modelo proposto é considerada como ortotrópica, possuindo diferentes propriedades mecânicas nas três direções principais. Para a direção longitudinal (L) adotou-se a direção 3 (ou z) do eixo do SAP; direção radial (R) que adotou-se como direção 1 (ou $\mathrm{x}$ ) e direção tangencial (T) que se adotou como direção 2 (ou y). Para cada direção considerada existe um módulo de elasticidade e também um módulo de elasticidade transversal (que agem entre os planos).

Surgem, devido à ortotropicidade, coeficientes de Poisson diferentes, pois a relação entre os planos não é isotrópica. Por exemplo, aplicando-se uma tensão na direção 3 , considerando a superposição de efeitos devido à linearidade, surgem deformações nessa própria direção e também nas direções 1 e 2 . Mas em cada direção têm-se comportamentos mecânicos diferentes; as deformações nas direções 1 e 2 têm proporcionalidades diferentes entre elas. Exemplificando numericamente: para uma tensão aplicada na direção 3: $-\sigma_{33}$ a deformação nesta direção é $-\varepsilon_{33}$ enquanto na direção 2 é: $+v_{13}$. $\varepsilon_{33}$ e na direção 1 é $+v_{23} . \varepsilon_{33}$. Caso o material fosse isotrópico ter-se-ia uma deformação na direção 3 de $-\varepsilon_{33}$ e nas outras duas $+v . \varepsilon_{33}$.

Como se deseja realizar uma modelagem teórica para o corpo - de - prova de madeira, observou-se que alguns autores descrevem as relações entre as propriedades mecânicas.

Bodig e Jayne (1982) sugerem as seguintes relações entre constantes elásticas da madeira: $\mathrm{E}_{\mathrm{L}}: \mathrm{E}_{\mathrm{R}}: \mathrm{E}_{\mathrm{T}} \approx 20: 1,6: 1 ; \mathrm{G}_{\mathrm{LR}}: \mathrm{G}_{\mathrm{LT}}: \mathrm{G}_{\mathrm{RT}} \approx 10: 9,4: 1 ; \mathrm{E}_{\mathrm{L}}: \mathrm{G}_{\mathrm{LR}} \approx 14: 1$. Esses autores apresentam os valores médios dos coeficientes de Poisson para coníferas e folhosas (Tabela 11).

Tabela 11 - Média dos coeficientes de Poisson.

\begin{tabular}{c|c|c}
\hline Índice & Coníferas & Folhosas \\
\hline$v_{\mathrm{LR}}$ & 0,37 & 0,37 \\
$v_{\mathrm{LT}}$ & 0,42 & 0,50 \\
$v_{\mathrm{RT}}$ & 0,47 & 0,67 \\
$v_{\mathrm{TR}}$ & 0,35 & 0,33 \\
$v_{\mathrm{RL}}$ & 0,041 & 0,044 \\
$v_{\mathrm{TL}}$ & 0,033 & 0,027 \\
\hline
\end{tabular}

Em termos de Brasil encontra-se na literatura dois interessantes trabalhos: Mascia (1991) que apresentou relações similares para algumas madeiras,tais como: Guapuruvú, Ipê, Angico e Pinus, e Ballarin and Nogueira (2003) para o Eucalipto citriodora.

No SAP2000 existe a possibilidade de adotar um material com características ortotrópicas, sendo assim, criou-se o material chamado de Pinus Elliotti.

Optou-se pela análise linear, pois a madeira possui um comportamento elástico-linear, valendo a superposição de efeitos nas relações constitutivas. 
Nessa simulação (Figura 7), foram considerados os pares de atuação das forças aplicadas. A convenção de tensão no SAP 2000 é a seguinte: direção 1: corresponde à direção $\mathrm{X}$ apresentada no modelo; direção 2: corresponde à direção $\mathrm{Y}$ apresentada no modelo; direção 3: corresponde à direção $\mathrm{Z}$ apresentada no modelo.

Como o carregamento atua simultaneamente nas direções 1 e 3 , optou-se por criar carregamentos independentes e depois combiná-los. Assim foram consideradas diversas combinações. Para à direção 3 aplicou-se uma tensão de $2,5 \mathrm{kN} / \mathrm{m}^{2}$. Para a direção 1 aplicou-se uma de $0,202 \mathrm{kN} / \mathrm{m}^{2}$. Os valores das pressões foram obtidos dos ensaios biaxiais de compressão.

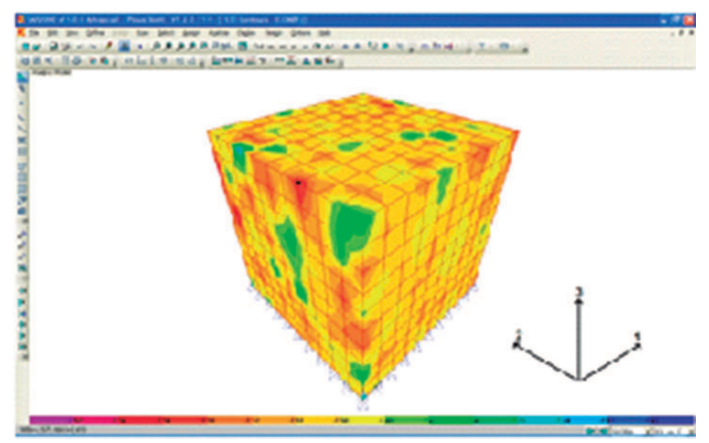

a) Tensão $\sigma_{33}$ em KN/m².

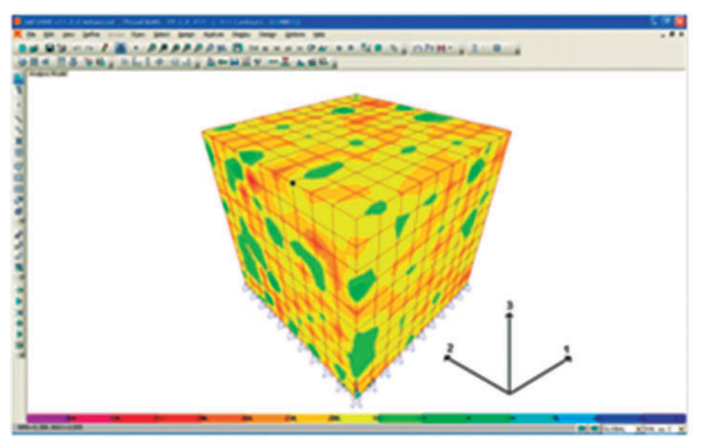

b) Tensão $\sigma_{11}$ em KN/m².

Figura 7- Resultados da combinação.

\subsection{Análise do Círculo de Mohr}

Para cada combinação adotou-se um determinado ponto sendo preferencialmente adotados pontos que apresentaram os maiores valores de tensões. Observa-se que aqui não se segue a convenção usual $\sigma_{1}>\sigma_{2}>\sigma_{3}$ para o desenho do círculo de Mohr (Chen and Salleb,1982), Figura 8. Assim, para a análise de tensões tem-se as tensões principais encontradas nesse ponto correspondente ao círculo de Mohr foram as seguintes, considerando a convenção adotada no SAP2000: $\sigma_{33}=-25,60 \mathrm{MPa} ; \sigma_{11}=-2,08 \mathrm{MPa}$; $\sigma_{22}=+0,22 \mathrm{MPa}$. 


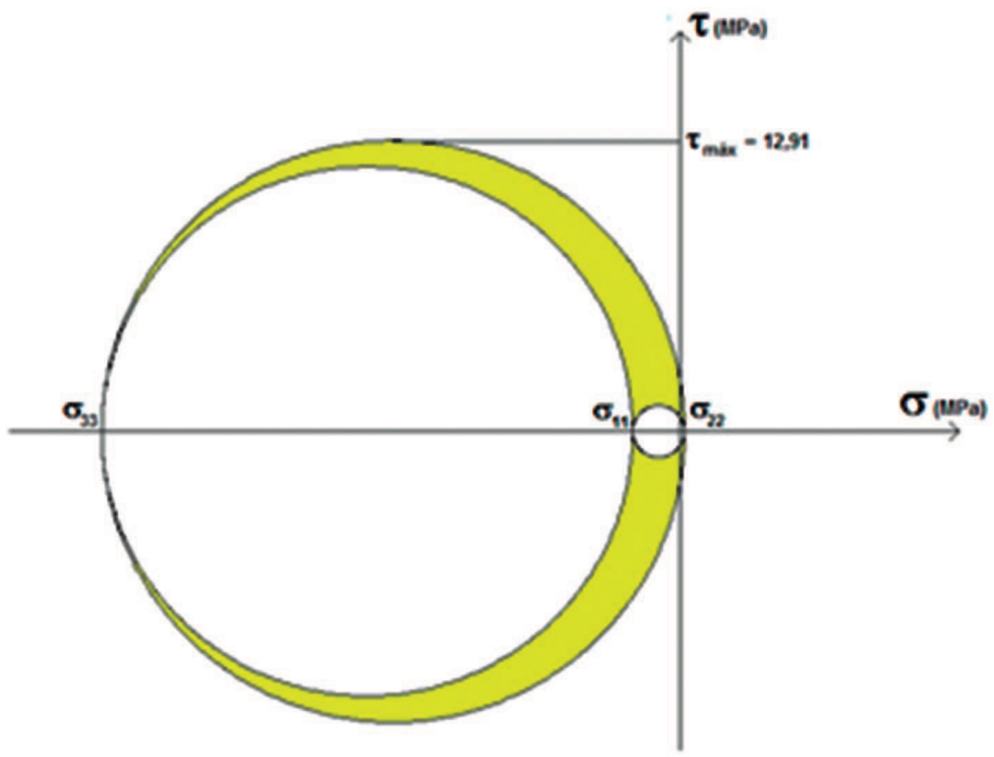

Figura 8 - Círculo de Mohr para um ponto com as máximas tensões.

\subsection{Comentários dos Resultados obtidos}

Todos os valores das faces do corpo - de - prova apresentaram dispersões nos resultados, pois são zonas de concentrações de tensões. Isso pode ser observado principalmente na região onde foram definidos os apoios do elemento, sendo que esses sustentam apenas os quatro pontos da face de cada cubo pequeno, o que seria muito diferente se o elemento estivesse dividido em infinitas partes. Mas devido à limitação de hardware e também de processamento, optou-se pela configuração atual.

Observando todas as combinações, verifica-se que as tensões $\sigma_{22}$ apresentam valores positivos de tração. Esses valores já eram esperados, pois o corpo - de - prova só recebe carga de compressão nas direções 1 e 3 e que, pelas relações constitutivas do material, a direção 2 só poderia apresentar uma expansão, confirmando o exemplo citado anteriormente.

Em geral as tensões $\sigma_{11}$ e $\sigma_{33}$ de todas as combinações apresentaram aumento de valores. As tensões $\sigma_{33}$, de maiores intensidades aplicadas, apresentaram uma distribuição mais uniforme ao longo do elemento estudado, com os maiores valores na região central do corpo-de-prova.

As tensões $\sigma_{11}$ apresentaram, em grande parte do elemento, valores menores do que aqueles aplicados na direção 1 . Essa diminuição é conseqüência do grande valor aplicado na direção 3 em relação ao da direção 1 . Pela mesma idéia de contração e expansão das outras direções é que essa diminuição é visível. Quando se aplica uma carga de valor alto na direção 3 , tem-se uma grande deformação negativa (contração) 
na mesma direção e para as outras se tem uma expansão da ordem de $+v_{\mathrm{ij}}$. $\varepsilon_{33}$. Na direção 1 tem-se uma carga menor em torno de 4 vezes a vertical, logo, a deformação nessa direção será $-\varepsilon_{11}+v_{\mathrm{ij}}$. $\varepsilon_{33}$. É claro que o valor $+v_{\mathrm{ij}} \cdot \varepsilon_{33}$ não é predominante nessa direção, pois depende do valor do coeficiente Poisson, mas acarreta uma diminuição expressiva da deformação na direção 1 , diminuindo consequentemente as tensões $\sigma_{11}$, fato observado na modelagem.

Através da análise dos gráficos que representam os círculos de Mohr, verifica-se que existe a influência da tensão de cisalhamento que também é responsável pela ruptura do material. Isso comprova a validade do critério de Tsai-Wu onde a ruptura é causada tanto pelas tensões normais quanto pelas tangenciais.

\section{Conclusões}

O trabalho desenvolvido teve como objetivo geral o estudo de critérios de resistência e suas teorias para a aplicação nos estudos do material a ser analisado. Nota-se que cada material possui características de resistência e que são analisados por determinados critérios de resistência que levam em consideração seu comportamento elástico em diferentes direções.

Para os principais materiais que são utilizados na construção civil (madeira, aço, concreto) foram desenvolvidas e estão sendo desenvolvidas teorias que explicam e condizem com o comportamento mecânico de cada um. Mais especificamente, essa pesquisa teve como objetivo a aplicação do critério de Tsai-Wu, adequado para materiais anisotrópicos, à madeira, a qual é um material ortotrópico e tem comportamento mecânico de suas propriedades completamente diferente do comportamento das propriedades do aço (material isotrópico) e do concreto.

Pela análise dos resultados obtidos no ensaio uniaxial de compressão e os ensaios biaxiais de compressão, verificou-se que o critério de Tsai-Wu apresentou, em média, resultados satisfatórios e coerentes. Ao se utilizar o critério de Tsai-Wu, para o estado plano de tensões múltiplas, observou-se a grande influência do coeficiente de interação $\mathrm{F}_{12}$ nas estimativas de resistência, principalmente nos ensaios biaxiais de compressão. Fato também observado por Nicolas (2006. Este conclui que para estados múltiplos de tensões, há necessidade de utilização do critério de Tsai-Wu que leva em consideração a interação entre as tensões.

Através do estudo desenvolvido verificou-se a sensibilidade do fator $\mathrm{F}_{12}$, pois ao se fixarem os valores limites e substituí-los nas equações do critério uma pequena alteração no valor do coeficiente $\mathrm{F}_{12}$ provoca alterações significativas nas estimativas de resistência. Logo o critério de Tsai-Wu foi satisfatório considerando-se toda a heterogeneidade da madeira e as dificuldades associadas a uma análise experimental com um equipamento de testes biaxial.

Através das curvas obtidas da espécie Pinus elliottii, verificou-se que cada curva possui um grupo de pontos que estão em suas proximidades. As curvas mais adequa- 
das correspondem respectivamente aos valores de 5,013 $\times 10^{-3}$ e $1,572 \times 10^{-3} \mathrm{MPa}^{-2} \mathrm{para} \mathrm{F}_{12}$ como mostrado na Figura 4. Através das curvas obtidas da espécie Goupia glabra, foi possível uma análise e verificação mais adequada dos resultados, tendo em vista um número de corpos-de-prova ensaiados maior. As melhores curvas correspondem respectivamente aos valores de: $9,7 \times 10^{-5}, 2,6 \times 10^{-5} \mathrm{MPa}^{-2}$ e 0 para $\mathrm{F}_{12}$ conforme a Figura 5 .

Também se conclui que o coeficiente adotado por Cowin (1979), $F_{12}=\sqrt{F_{11} \cdot F_{22}}-\frac{1}{2 \cdot f_{v}^{2}}$, foi o que apresentou resultados mais próximos aos valores dos ensaios biaxiais.

Assim, considerando-se ensaios biaxiais de compressão, para obtenção do melhor critério, observou-se que os menores pares de valores (em módulo) das tensões aplicadas verticalmente e horizontalmente podem definir o parâmetro $\mathrm{F}_{12}$, verificando-se sua grande sensibilidade quanto às variações das tensões aplicadas nos corpos-de-prova. Vale ressaltar que um dos problemas enfrentados nos ensaios biaxiais dos corpos-de-prova foi manter a calibração do equipamento de compressão axial (alicate).

Observa-se, também, que na confecção dos corpos-de-prova foram verificados os ângulos de inclinação das fibras, assim como o controle de umidade, e de temperatura $\left(20^{\circ} \mathrm{C}\right)$. Concluiu-se, então, que as causas principais das dispersões podem estar relacionadas com a heterogeneidade do material nas direções ortotrópicas (falhas pré-existentes de constituição) e possíveis defeitos provenientes da variação dimensional após a elaboração dos corpos-de-prova.

Observa-se, ainda, que um fator de grande influência no sucesso da pesquisa e na modelagem via SAP 2000 foi a utilização das espécies de madeira Pinus elliottii e Goupia glabra, madeiras essas que apresentam características mecânicas homogêneas em cada direção ortotrópica (radial, tangencial e longitudinal).

Por fim destaca-se que, a formulação de ensaios biaxiais para diversos materiais pode ser muito útil para o desenvolvimento de novas tecnologias e também para otimização de estruturas. Os principais fatores que definem o arranjo para esse tipo de ensaio são: tipo de material, dificuldades na montagem do ensaio, sua execução e interpretação dos resultados. Especificamente, para à madeira e as estruturas de madeira, o estudo do critério de Tsai-Wu é importante, pois desse estudo advém um melhor entendimento de suas propriedades elásticas e de resistência, as quais contribuem para formulações mais adequadas e completas no campo da mecânica estrutural.

Para futuras pesquisas nessa área, sugere-se a realizar os ensaios biaxiais com um número maior de espécies de madeira para uma interpretação mais geral de resultados e desse modo, uma definição mais específica dos parâmetros envolvidos no critério de resistência de Tsai-Wu.

\section{Agradecimentos}

Os autores agradecem à Fapesp (Projeto n. 2004/15481-5) e ao CNPq (Projeto n.301504/2004-0) pelo apoio no desenvolvimento dessa pesquisa. 


\section{Referências}

ABNT - Associação Brasileira de Normas Técnicas. (1997) NBR 7190: Projeto de Estruturas de Madeira. Rio de Janeiro: ABNT.

Alvin, R. A. A. (2003).Avaliação Experimental de propriedades Mecânicas dos tecidos estruturais. Dissertação (mestrado), Escola Politécnica, USP, São Paulo.

Ballarin, A. W., Nogueira, M. (2003) Caracterização elástica da madeira de Eucalyptus citriodora. Cerne, http://www.dcf.ufla.br, v. 9, n. 1, p. 69-83.

Bodig, J., Jayne B. A. (1982) Mechanics of Wood and Wood Composites. New York: Van Renhold. Bibliografia: p. 291-302.

Chen, W. F., Saleeb, A. (1982) Constitutive equations for engineering materials. New York: John Wiley \& Sons, V. 1: Elasticity and Modeling, p1-181.

Cowin, S. C. (1979) On the strength anisotropic of bone and wood. Journal of Applied Mechanics, v.46, p.832-838.

Eberhardsteiner, J. (2002) Mechanisches Verhalten von Fichtenholz - Experimentelle Bestimmung der biaxialen Festigkeitseigenschaften. Springer, in German.

Hasebe, K., Usuki, S. Application of orthotropic failure criterion to wood. Journal of Engineering Mechanics, n. 4, v.115, p.867-872. Apr. 1989.Hoffman, O. (1967)The brittle strength of ortotropic materials. Journal of Composite Materials, n. 2, v.1, p.200-206.

Hoffman, O. The brittle strength of ortotropic materials. Journal of Composite Materials, n. 2, v.1, p.200-206. 1967.

Ferreira,O.P. (2003) Madeira: Uso Sustentável na Construção Civil. IPT. Instituto de Pesquisas Tecnológicas: SVMA: SINDUSCON-SP. São Paulo, 57p.

Liu, J. Y. (1984) Evaluation of the tensor polynomial strength theory for wood. Journal of Composite Material, v. 18, p. 216-226.

Mascia, N.T. (1991) Considerações à respeito da anisotropia na madeira. São Carlos, Tese de Doutorado - Escola de Engenharia de São Carlos/USP, 295p.

Mascia, N. T., Nicolas, E. A., Todeschini, R. (2007) Evaluation of off-axis wood compression strength In: International Conference on Experimental Mechanics, Alexandroupolis: European Association for Experimental Mechanics (EURASEM), v. 1. p. 1-8.

Mascia, N. T., Nicolas, E. A., Sverzut, C. B. (2007) Ensaios Biaxiais e a Determinação de Parâmetros de Resistência da Madeira In: 9a. Coteq - Conferência Internacional sobre Tecnologia de Equipamentos, Salvador: Abende, v. 1. p. 1-17.

Nicolas, E. A. Critérios de Resistência de Materiais Anisotrópicos Aplicados à Madeira. Tese (Doutorado), Faculdade de Engenharia Civil, UNICAMP, Campinas, 289f, 2006.

SAP 2000 (2010)Versão 7.0, Berkeley, California: Computers and Structures, Inc.

Suhling, J. C. (1985) Constitutive relations and failure predictions for nonlinear orthotropic media. Pennsylvania: Tese de Doutorado, University of Wisconsin.

Todeschini, R., Mascia, N. T., Nicolas, E. A. (2008) Ensaios Biaxiais e Critérios de Resistência de Materiais Anisotrópicos Aplicados à Madeira In: XI Encontro Brasileiro em Madeiras e Estruturas de Madeira, Londrina. p. 1-15.

Tsai, S. W. , Wu, E. M. (1971) A general theory of strength for anisotropic materials. Journal of Composite Materials. New York: n. 1, v. 5, p. 58-80. 


\title{
Evaluation of an anisotropic criterion applied to wood using uniaxial and biaxial tests
}

\begin{abstract}
The development of the uniaxial and biaxial tests permits a better understanding of the mechanical behavior of a material that has main strength directions and it is undoubtedly a method that researchers identified to achieve consistent results with the actual situation that a specific structure presents. This study aimed at studying the strength criterion proposed by Tsai and $\mathrm{Wu}$, performing uniaxial and biaxial tests. The evaluation of this criterion was restricted to compression biaxial. With a compression biaxial test, the stresses in two main directions were carried out simultaneously, using a standard equipment test for one direction and for the perpendicular direction an equipment type pliers especially developed for this purpose. The failure parameter studied was $\mathrm{F}_{12}$, which evaluates the possibility of failure surface to be opened or closed, also considering that for the safety of design it is essential to obtain a closed surface for the failure criterion adopted. Furthermore, it was noted that in the compression biaxial test the parameter $\mathrm{F}_{12}$ was obtained directly. For the species of wood Pinus elliottii and Goupia glabra,the obtained experimental values were satisfactory with some inconsistencies observed in the light of the heterogeneity of the material.
\end{abstract}

Keywords: Failure Criterion,Tsai-Wu Criterion, Uniaxial and Biaxial Tests.

\section{Tsai-Wu Failure Criterion}

The main objective of a failure criterion is to interpret the combined loadings and multiaxial stresses that eventually lead to failure. For instance, whereas the safety of a tensile bar is simply defined by comparing its strength as determined in tension tests with current stresses, a specific criterion would be necessary to predict the safety of a structure subject to complex loadings or multiaxial states of stress. In addition, it would be very inconvenient to perform experimental tests for each specific state of stress to establish this criterion. For these cases, a failure criterion should take into consideration a limited number of material parameters to determine failure properties.

In order to establish a failure criterion, specific procedures must be followed. First, one identifies the phenomenon responsible for the failure after several mechanical analyses of possible combinations of loadings. Then the validity of the criterion is verified by comparing real behavior with complex combinations of loadings. This implies that the establishment of a unique criterion is incoherent due to the variety of materials used in engineering, evidently with different mechanical behavior.

According to Bodig and Jayne (1982), for isotropic and homogeneous materials, failure criteria were developed that displayed a linear mechanical behavior, that is, a linear stress-strain relationship until failure. Consequently, when these criteria are 
applied to wood, the results are inadequate because the nonhomogeneity and nonelastic behavior of the material need to be considered.

As stated, Tsai and Wu (1971) developed a general failure criterion to evaluate anisotropic materials. This criterion may be expressed in terms of strength tensors in polynomial form as presented by Equação (1):

$$
\mathrm{F}_{\mathrm{i}} \cdot \sigma_{\mathrm{i}}+\mathrm{F}_{\mathrm{ii}} \cdot \sigma_{\mathrm{i}} \cdot \sigma_{\mathrm{i}}=1 \quad(\mathrm{i}, \mathrm{j}=1,2, \ldots, 6)
$$

where $F_{i}$ and $F_{i j}$ represent the strength tensors of second and fourth rank, respectively, following the contracted notation, and the $o_{i}$ and ó ${ }_{j}$ represent stresses.

Considering now an orthotropic material under a plane stress field in which axes 1 and 2 are the principal directions, Equation (1) can be expanded and written as

$$
\mathrm{F}_{1} \cdot \sigma_{1}+\mathrm{F}_{2} \cdot \sigma_{2}+\mathrm{F}_{11} \cdot \sigma_{1}^{2}+\mathrm{F}_{22} \cdot \sigma_{2}^{2}+2 \mathrm{~F}_{12} \cdot \sigma_{1} \cdot \sigma_{2}+\mathrm{F}_{44} \cdot \sigma_{4}^{2}=1
$$

where $\sigma_{1}$ and $\sigma_{2}$ are the normal stresses, $\sigma_{4}$ is the shear stress. Table 1 lists the expressions to calculate the strength tensors of second $\left(\mathrm{F}_{1}, \mathrm{~F}_{2}\right)$ and fourth rank $\left(\mathrm{F}_{11}, \mathrm{~F}_{22}, \mathrm{~F}_{44}\right.$, and $F_{12}$ ). All these terms, except $F_{12}$, are determined via the results of compression, tension, and shear tests. The strength coefficient or coefficient of interaction $\mathrm{F}_{12}$ a priori requires biaxial tests, as can be seen in the works of Eberhardsteiner (2002), for instance.

Table 1 - Strength Coefficients.

\begin{tabular}{c|c|c}
\hline $\mathrm{F}_{1}=\frac{1}{\mathrm{f}_{\mathrm{t} 1}}-\frac{1}{\mathrm{f}_{\mathrm{c} 1}}$ & $\mathrm{~F}_{2}=\frac{1}{\mathrm{f}_{\mathrm{t} 2}}-\frac{1}{\mathrm{f}_{\mathrm{c} 2}}$ & $\mathrm{~F}_{44}=\frac{1}{\mathrm{f}_{\mathrm{v} 4}^{2}}$ \\
\hline $\mathrm{F}_{11}=\frac{1}{\mathrm{f}_{\mathrm{t} 1} \cdot \mathrm{f}_{\mathrm{cl}}}$ & $\mathrm{F}_{22}=\frac{1}{\mathrm{f}_{\mathrm{t} 2} \cdot \mathrm{f}_{\mathrm{c} 2}}$ & $\mathrm{~F}_{12}< \pm\left|\sqrt{\mathrm{F}_{11} \cdot \mathrm{F}_{22}}\right|$ \\
\hline
\end{tabular}

In Table $1, f_{t 1}$ is the tensile strength along the 1-axis, $f_{c 1}$ is the compressive strength along the 1-axis, $\mathrm{f}_{\mathrm{t} 2}$ is the tensile strength along the 2 -axis, $\mathrm{f}_{\mathrm{c} 2}$ is the compressive strength along the 2-axis and $\mathrm{f}_{\mathrm{v} 4}$ is the shear strength along the 1-2 plane.

In addition, according to Tsai and $\mathrm{Wu}$ ((1971) certain stability conditions are associated with the strength tensors and the magnitudes of the interaction terms are related to the following inequality: $-\sqrt{\mathrm{F}_{11} \cdot \mathrm{F}_{22}}<\mathrm{F}_{12}<\sqrt{\mathrm{F}_{11} \cdot \mathrm{F}_{22}}$. Geometrically, this inequality ensures that the failure surface will be ellipsoidal in a three-dimensional stress field and elliptical in a plane field. The shape of the surface cannot be open ended like a hyperboloid or hyperbole. In Table 1 , it is assumed that $\mathrm{F}_{12}$ is in the range expressed by the above inequality.

In this context, this paper aims to compare experimental results of biaxial compression tests with the Tsai-Wu criterion. To achieve this, compression, tension, and 
shear tests as well as biaxial compression tests were performed. As a consequence this research intends to contribute with the existing literature on failure analysis specially showing aspects of the mechanical behavior of the tropical wood species Pinus elliottii and Goupia glabra .

\section{Biaxial Compression Tests}

A special equipment was developed to carry out the biaxial compression tests and it can be seen in Figure 1 (Nicolas, 2006). This equipment was connected with a universal test machine in which vertical and horizontal loads were applied to the surface of the specimen to achieve a biaxial stress field. A plate and a sphere simulating a hinged connection were used to uniformly distribute the horizontal load.

The cubic specimen adopted in this research was $40 \mathrm{~mm}$ and the species, specific gravity, and moisture content were the same as in the uniaxial tests. A total of 62 specimens were tested.
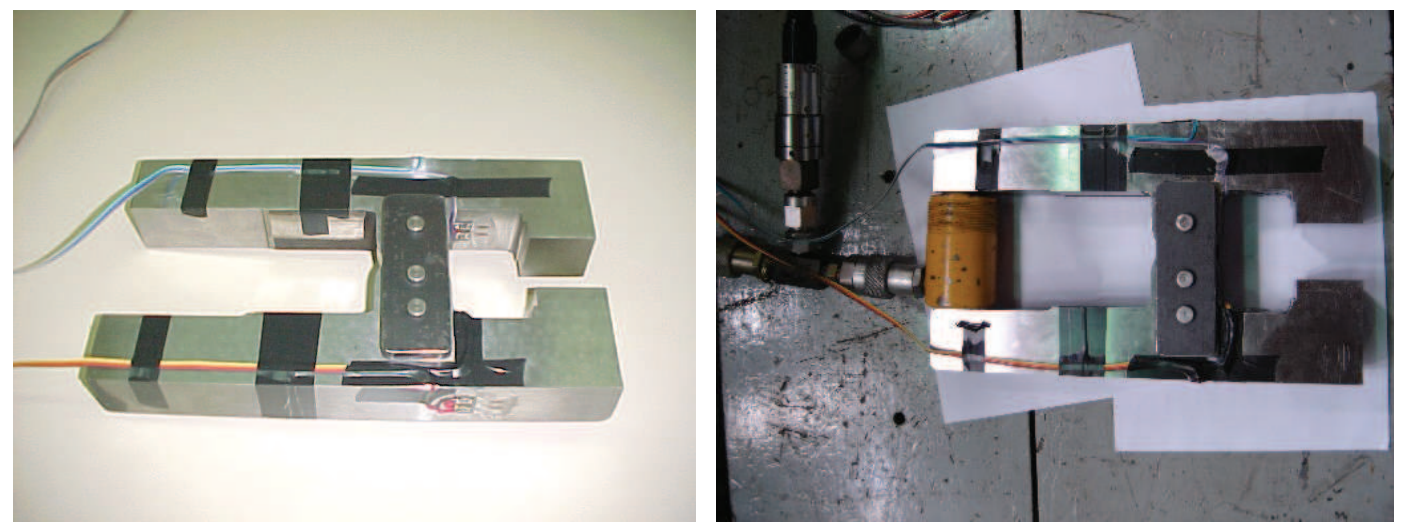

Figure 1 - Equipment for biaxial test.

\section{Discussion of results}

By analyzing the test results of uniaxial compression and biaxial compression tests, it was noted that the Tsai-Wu criterion can be considered, on average, satisfactory and consistent. When using the Tsai-Wu criterion for the state plane for multiple stresses, the great influence of the interaction coefficient in the prediction of resistance, especially in biaxial compression tests is observed. Also as was observed by Mascia, Nicolas and Todeschini (2007), Tsai-Wu criteriont takes into account the interaction between the stresses.

The obtained results showed that $\mathrm{F}_{12}$ is a very sensitivity factor. A small change in the value of the coefficient causes significant changes in estimates of resistance, so the criterion of Tsai- Wu was considered satisfactory. 
From the data of Pinus elliottii, it was found that each curve had a group of results that were near the curve from the Tsai-Wu criterion. The best curves correspond to the values of $5,013 \times 10^{-3}$ and $1,572 \times 10^{-3} \mathrm{MPa}^{-2}$ for $\mathrm{F}_{12}$. For the species Goupia glabra, there was a better result, since the number of specimens used in evidence tested was higher. The best curve corresponds to the values of $9,7 \times 10^{-5}$ and $2,6 \times 10^{-5}$ and 0 for $\mathrm{F}_{12}$.

It was also concluded that the rate adopted by Cowin (1979), i.e., $F_{12}=\sqrt{F_{11} \cdot F_{22}}-\frac{1}{2 \cdot f_{v}^{2}}$ presented similar results to the values of biaxial tests.

Thus, considering biaxial compression tests to obtain the best criterion, it was observed that the smallest pairs of stresses values, which were applied vertically and horizontally, can define the values of the parameter $F_{D}$, verifying its high sensitivity for variations of stresses applied on the specimens of the tests. It is noteworthy that one of the problems found in the biaxial tests was to maintain equipment calibration axial compression (pliers).

\section{Conclusions}

A comparison between the experimental results from biaxial compression tests of a species of Brazilian wood and the Tsai-Wu failure criterion, adequate for anisotropic materials and multiaxial stress cases, was carried out.

To predict failure, the main difficulty of the Tsai-Wu criterion application, discussed in much of the technical literature, is to determine the coefficient of interaction $\mathrm{F}_{12}$. This difficulty is due to several formulas, proposed by different researchers, involving uniaxial or biaxial experimental procedures and the difficulties in analyzing sensitivity from the experimental results. In this study, a special equipment was constructed to conduct two-dimensional loads to evaluate $\mathrm{F}_{12}$.

Basically, the values of the relationship $\mathrm{F}_{12} / \mathrm{F}_{12}$ limit $\mid$ that most closely matched the data from the biaxial tests were confined to the interval $0 \leq \mathrm{F}_{12} / \mathrm{F}_{12}$ limit $\mid \leq 0.2$. In general, the Tsai-Wu criterion exhibited satisfactory results in comparison with data from biaxial compression tests. This criterion presents a good approach when $\mathrm{F}_{12}=+$ limit. Already, the values for $\mathrm{F}_{12}$ proposed by Cowin generated failure envelopes closer to the experimental data, with the advantage of requiring the results from uniaxial tests only. Additionally, Cowin's $\mathrm{F}_{12}$ generated the closest fit to the values from the biaxial tests.

For future research in this area, it is suggested to perform the biaxial tests with a larger number of species of wood for a general interpretation of results and thus a more specific definition of the parameters that involved in the failure criterion of Tsai-Wu. 


\section{References}

Bodig, J., Jayne B. A. (1982) Mechanics of Wood and Wood Composites. New York: Van Renhold. Bibliografia: p. 291-302.

Cowin, S. C. (1979) On the strength anisotropic of bone and wood. Journal of Applied Mechanics, v.46, p.832-838.

Eberhardsteiner, J. (2002) Mechanisches Verhalten von Fichtenholz - Experimentelle Bestimmung der biaxialen Festigkeitseigenschaften. Springer, in German.

Mascia, N. T., Nicolas, E. A., Todeschini, R. (2007) Evaluation of off-axis wood compression strength In: International Conference on Experimental Mechanics, Alexandroupolis: European Association for Experimental Mechanics (EURASEM), v.1. p.1 - 8.

Nicolas, E. A. Critérios de Resistência de Materiais Anisotrópicos Aplicados à Madeira. Tese (Doutorado), Faculdade de Engenharia Civil, UNICAMP, Campinas, 289f, 2006.

SAP 2000 (2010) Versão 7.0, Berkeley, California: Computers and Structures, Inc.

Tsai, S. W. , Wu, E. M. (1971) A general theory of strength for anisotropic materials. Journal of Composite Materials. New York: n. 1, v.5, p.58-80.

\section{Acknowledgements}

The authors gratefully acknowledge CNPq (n. 301504/2004-0) and FAPESP (n. 2004/15481-5), Brazilian Foundations, for the financial supporting of this research. 